\title{
On the mathematical model of Rabies by using the fractional Caputo-Fabrizio derivative
}

\author{
Seher Melike Aydogan', Dumitru Baleanu²,3, Hakimeh Mohammadi and Shahram Rezapour ${ }^{5,6,7^{*}}$ (D)
}

\author{
"Correspondence: \\ shahramrezapour@duytan.edu.vn; \\ sh.rezapour@mail.cmuh.org.tw; \\ rezapourshahram@yahoo.ca; \\ sh.rezapour@azaruniv.ac.ir \\ ${ }^{5}$ Institute of Research and \\ Development, Duy Tan University, \\ Da Nang, 550000, Vietnam \\ ${ }^{6}$ Faculty of Natural Sciences, Duy \\ Tan University, Da Nang, 550000, \\ Vietnam \\ Full list of author information is \\ available at the end of the article
}

\begin{abstract}
Using the fractional Caputo-Fabrizio derivative, we investigate a new version of the mathematical model of Rabies disease. Using fixed point results, we prove the existence of a unique solution. We calculate the equilibrium points and check the stability of solutions. We solve the equation by combining the Laplace transform and Adomian decomposition method. In numerical results, we investigate the effect of coefficients on the number of infected groups. We also examine the effect of derivation orders on the behavior of functions and make a comparison between the results of the integer-order derivative and the Caputo and Caputo-Fabrizio fractional-order derivatives.
\end{abstract}

MSC: Primary 34A08; 35A22; secondary 35A20

Keywords: Adomian decomposition method; Fixed point; Numerical simulation; Rabies model; The Caputo-Fabrizio fractional derivative

\section{Introduction}

The Rabies viral disease, which is characterized by the initial symptoms of fever and tingling at the site of exposure, causes inflammation of the brain in humans and other mammals. Another symptom of this disease is the inability to move parts of the body, harsh movements, uncontrolled excitement, fear of water, confusion, and loss of consciousness. After catching the disease, it usually takes one to three months for the initial symptoms to appear; at the same time, this period can take less than a week or more than a year, It depends on the distance the virus takes during the peripheral nerves to reach the central nervous system; however, this disease can often cause death [1]. Although it is possible to prevent Rabies, it still exists in groups of warm-blooded animals in more than 150 countries, and more than 55,000 people die of the disease each year. Rabies is still one of the most important issues in the field of human health and wildlife management [2-4]. There are various mathematical models explaining the release of Rabies by ordinary and partial differential equations (see, e.g., [5-7]). The study of disease dynamics is a dominating theme for many biologists and mathematicians (see, e.g., [8-12]). It has been studied by many researchers that fractional extensions of mathematical models of integer order rep-

(c) The Author(s) 2020. This article is licensed under a Creative Commons Attribution 4.0 International License, which permits use sharing, adaptation, distribution and reproduction in any medium or format, as long as you give appropriate credit to the original author(s) and the source, provide a link to the Creative Commons licence, and indicate if changes were made. The images or other third party material in this article are included in the article's Creative Commons licence, unless indicated otherwise in a credit line to the material. If material is not included in the article's Creative Commons licence and your intended use is not permitted by statutory regulation or exceeds the permitted use, you will need to obtain permission directly from the copyright holder. To view a copy of this licence, visit http://creativecommons.org/licenses/by/4.0/. 
resent the natural fact in a very systematic way such as in the approaches of Akbari et al. [13], Aydogan et al. [14, 15], Baleanu et al. [16-28], Ozdemir et al. [29-33], and Talaee et al. ([34]). Recently, many works related to the fractional Caputo-Fabrizio derivative [35] have been published (see, e.g., [8-12, 36-38], and [7]).

In this paper, we first study the integer-order Rabies model with the Caputo-Fabrizio fractional-order derivative and investigate the behavior of the results obtained from the model. We will also investigate the effect of the coefficients in the model on the number of infected animals.

Now we recall some fundamental notions. The Riemann-Liouville derivative of order $\eta$ for a function $f$ is given by

$$
D^{\eta} f(t)=\frac{d^{n}}{d t^{n}} \int_{0}^{t} \frac{f(s)}{(t-s)^{1-n+\eta}} d s, \quad n-1 \leq \eta<n
$$

The Caputo fractional derivative of order $\eta$ for a function $f$ via integrable differentiations is defined by

$$
{ }^{C} D^{\eta} f(t)=\frac{1}{\Gamma(n-\eta)} \int_{0}^{t} \frac{f^{(n)}(s)}{(t-s)^{\eta-n+1}} d s, \quad n=[\eta]+1 .
$$

Our second notion is a fractional derivative without singular kernel, introduced by Caputo and Fabrizio [35] in 2015. Let $b>a, f \in H^{1}(a, b)$, and $\eta \in(0,1)$. The Caputo-Fabrizio derivative of order $\eta$ for a function $f$ is defined by

$$
{ }^{C F} D^{\eta} f(t)=\frac{M(\eta)}{(1-\eta)} \int_{a}^{t} \exp \left(\frac{-\eta}{1-\eta}(t-s)\right) f^{\prime}(s) d s, \quad t \geq 0,
$$

where $M(\eta)$ is a normalization function such that $M(0)=M(1)=1$. If $f \notin H^{1}(a, b)$ and $0<\eta<1$, then this derivative can be presented for $f \in L^{1}(-\infty, b)$ as (see [39])

$$
{ }^{C F} D^{\eta} f(t)=\frac{\eta M(\eta)}{(1-\eta)} \int_{-\infty}^{b}(f(t)-f(s)) \exp \left(\frac{-\eta}{1-\eta}(t-s)\right) d s .
$$

Let $n \geq 1$ and $\eta \in(0,1)$. The fractional derivative ${ }^{C F} D^{\eta+n}$ of order $\eta+n$ is defined by ${ }^{C F} D^{\eta+n} f(t):={ }^{C F} D^{\eta}\left(D^{n} f(t)\right)$ [14]. The Laplace transform of the Caputo-Fabrizio derivative is defined by $L\left[{ }^{C F} D^{(\eta+n)} f(t)\right](s)=\frac{s^{n+1} L[f(t)]-s^{n} f(0)-s^{n-1} f^{\prime}(0)-\cdots-f^{(n)}(0)}{s+\eta(1-s)}$, where $0<\eta \leq 1$ and $M(\eta)=1$ [39]). The Riemann-Liouville fractional integral of order $\eta$ with $\operatorname{Re}(\eta)>0$ is defined by (see [14])

$$
I^{\eta} f(t)=\frac{1}{\Gamma(\eta)} \int_{0}^{t}(t-s)^{\eta-1} f(s) d s
$$

The Caputo-Fabrizio fractional integral is defined by (see [39])

$$
{ }^{C F} I^{\eta} f(t)=\frac{2(1-\eta)}{(2-\eta) M(\eta)} f(t)+\frac{2 \eta}{(2-\eta) M(\eta)} \int_{0}^{t} f(s) d s, \quad 0<\eta<1 .
$$

The Sumudu transform is derived from the classical Fourier integral [40-42]. Consider the set $A=\left\{F: \exists \lambda, k_{1}, k_{2} \geq 0,|F(t)|<\lambda \exp \left(\frac{t}{k_{j}}\right), \quad t \in(-1)^{j} \times[0, \infty)\right\}$. The Sumudu transform 
of a function $f \in A$ is defined by

$$
F(u)=S T[f(t) ; u]=\frac{1}{u} \int_{0}^{\infty} \exp (-t / u) f(t) d t\left[u \in\left(-k_{1}, k_{2}\right)\right]
$$

for all $t \geq 0$, and the inverse Sumudu transform of $F(u)$ is denoted by $f(t)=S T^{-1}[F(u)]$ [41]. The Sumudu transform of the Caputo derivative is given by

$$
S T\left[{ }^{c} D_{t}^{\eta} f(t) ; u\right]=u^{-\eta}\left[F(u)-\sum_{i=0}^{m} u^{\eta-i}\left[{ }^{c} D^{\eta-i} f(t)\right]_{t=0}\right],
$$

where $m-1<\eta \leq m$ [40]. Let $F$ be a function such that its Caputo-Fabrizio fractional derivation exists. The Sumudu transform of $F$ with Caputo-Fabrizio fractional derivative is defined by $S T\left({ }_{0}^{C F} D_{t}^{\eta}\right)(F(t))=\frac{M(\eta)}{1-\eta+\eta u}[S T(F(t))-F(0)]$ [43].

Let $(X, d)$ be a metric space. A map $g: X \rightarrow X$ is called a Picard operator if there exists $x^{*} \in X$ such that Fix $(g)=\left\{x^{*}\right\}$ and the sequence $\left(g^{n}\left(x_{0}\right)\right)_{n \in N}$ converges to $x^{*}$ for all $x_{0} \in X$ $([44])$.

\section{Mathematical model of Rabies with a fractional derivative involving a nonsingular kernel}

In 2014, Demirci [5] has investigated a model explaining the spread of Rabies with an integer order. In this section, we consider this model with a new approach of fractionalorder derivative. In this SIR model the total population of animals $N$ at time $t$ is divided into three groups, susceptible animals $S(t)$, infected animals $I(t)$, and recovered animals $R(t)$. Rabies is a fatal disease: the animals annually die from this disease, and the only way to control the mortality caused by this disease is vaccination. The desired model is as follows:

$$
\left\{\begin{array}{l}
\frac{d S}{d t}=b N+z(N-S-I)-d(N) S-a S-b I-c S I \\
\frac{d I}{d t}=b I+c S I-d(N) I-e I \\
\frac{d N}{d t}=N(b-d(N))-e I
\end{array}\right.
$$

where the parameters have the following meanings:

$b$ : the rate of per capita birth,

$z$ : waning immunity,

$d(N)$ : the rate function of per capita death, which depends on the population size $N$,

$a$ : the rate of vaccination,

$c$ : the rate of Rabies transmission,

$e$ : the mortality rate in disease.

The initial conditions are $S(0)=S_{0}, I(0)=I_{0}, N(0)=N_{0}$. In this model, $d(N)$ is a continuous increasing function on $R^{+}$such that $d(m)=b$ for some positive constant $m$.

In model (1), we are going to replace the integer-order derivative with the fractionalorder derivative introduced by Caputo and Fabrizio [35] as follows:

$$
\left\{\begin{array}{l}
{ }_{0}^{C F} D_{t}^{\eta} S(t)=b N(t)+z(N(t)-S(t)-I(t))-d(N) S(t)-a S(t)-b I(t)-c S(t) I(t), \\
C F D_{t}^{\eta} I(t)=b I(t)+c S(t) I(t)-d(N) I(t)-e I(t), \\
{ }_{0}^{C F} D_{t}^{\eta} N(t)=N(t)(b-d(N))-e I(t),
\end{array}\right.
$$


with initial conditions $S(0)=S_{0}, I(0)=I_{0}$, and $N(0)=N_{0}$. In system (2) the right-hand sides of the equations have dimension time $e^{-1}$. When we change the order of the equations to $\eta$, the dimension of the left-hand side becomes time ${ }^{-\eta}$. In order to make the dimensions match, we should change the dimensions of the parameters, and the system we obtain eventually is

$$
\left\{\begin{array}{l}
{ }_{0}^{C F} D_{t}^{\eta} S(t)=b^{\eta} N(t)+z^{\eta}(N(t)-S(t)-I(t))-d^{\eta}(N) S(t)-a^{\eta} S(t)-b^{\eta} I(t)-c^{\eta} S(t) I(t), \\
{ }_{0}^{C F} D_{t}^{\eta} I(t)=b^{\eta} I(t)+c^{\eta} S(t) I(t)-d^{\eta}(N) I(t)-e^{\eta} I(t) \\
{ }_{0}^{C F} D_{t}^{\eta} N(t)=N(t)\left(b^{\eta}-d^{\eta}(N)\right)-e^{\eta} I(t)
\end{array}\right.
$$

For simplicity, we consider $A=a^{\eta}, B=b^{\eta}, C=c^{\eta}, D(N)=d^{\eta}(N), E=e^{\eta}$, and $Z=z^{\eta}$, and we use $D$ instead of $D(N)$. We also assume that there exists a positive constant $M$ such that $D(M)=B$. Then

$$
\left\{\begin{array}{l}
{ }_{0}^{C F} D_{t}^{\eta} S(t)=B N(t)+Z(N(t)-S(t)-I(t))-D S(t)-A S(t)-B I(t)-C S(t) I(t) \\
{ }_{0}^{C F} D_{t}^{\eta} I(t)=B I(t)+C S(t) I(t)-D I(t)-E I(t) \\
{ }_{0}^{C F} D_{t}^{\eta} N(t)=N(t)(B-D)-E I(t)
\end{array}\right.
$$

where $0<\eta \leq 1, S(0)=S_{0}, I(0)=I_{0}$, and $N(0)=N_{0}$. In the next section, we show that system (3) has a unique solution.

\section{Equilibrium points of the model and asymptotic stability}

We set the right-hand side of the equations to zero to determine the equilibrium points of the fractional-order system (3):

$$
{ }^{C F} D_{t}^{\eta} S(t)={ }^{C F} D_{t}^{\eta} I(t)={ }^{C F} D_{t}^{\eta} N(t)=0
$$

By solving these algebraic equations we obtain the equilibrium points $E^{0}=\left(\frac{(B+Z) M}{B+Z+A}, 0, M\right)$ and $E^{*}=\left(S^{*}, I^{*}, N^{*}\right)$ such that

$$
S^{*}=\frac{D+E-B}{C}, \quad I^{*}=\frac{(A+D+Z)(B-D)}{C(D+Z)}, \quad N^{*}=\frac{E(A+D+Z)}{C(D+Z)} .
$$

Also, by the next-generation method [45] we obtain the basic reproduction number $R_{0}=$ $\frac{C M(B+Z)}{E(A+B+Z)}$. To investigate the stability of equilibrium point, we first consider the fractionalorder linear system

$$
{ }^{C F} D_{t}^{\eta} v(t)=\psi v(t)
$$

where $v(t) \in R^{n}, \psi \in R^{n \times n}$, and $0<\eta<1$.

Definition 3.1 [46] For system (4) with Caputo-Fabrizio fractional derivative, the characteristic equation is given by

$$
\operatorname{det}(\lambda(I-(1-\eta) \psi)-\eta \psi)=0
$$


The Jacobian matrix associated with system (3) is

$$
J=\left[\begin{array}{ccc}
-(Z+D+A+C I) & -(Z+B+C S) & B+Z \\
C I & B+C S-D-E & 0 \\
0 & -E & B-D
\end{array}\right]
$$

and thus the characteristic equation of system (3) is

$$
\operatorname{det}(\lambda(I-(1-\eta) J)-\eta J)=0 .
$$

Since $E^{0}=\left(\frac{(B+Z) M}{B+Z+A}, 0, M\right)$, we have $N=M$, so that $D(N)=D(M)=B$. The Jacobian matrix associated with system (3) in $E^{0}$ is

$$
J\left(E^{0}\right)=\left[\begin{array}{ccc}
-(Z+B+A) & -Z-B-\frac{C M(B+Z)}{A+B+Z} & B+Z \\
0 & -E+\frac{C M(B+Z)}{A+B+Z} & 0 \\
0 & -E & 0
\end{array}\right]
$$

and thus the characteristic equation is

$$
\begin{aligned}
\lambda & {[\lambda(1+(1-\eta)(A+B+Z))+\eta(A+B+Z)]\left[\lambda\left(1-(1-\eta)\left(-E+\frac{C M(B+Z)}{A+B+Z}\right)\right)\right.} \\
& \left.-\eta\left(-E+\frac{C M(B+Z)}{A+B+Z}\right)\right]=0 .
\end{aligned}
$$

The roots of the latter are as follows:

$$
\lambda_{1}=0, \quad \lambda_{2}=\frac{-\eta(A+B+Z)}{1+(1-\eta)(A+B+Z)}, \quad \lambda_{3}=\frac{\eta\left(\frac{-E(A+B+Z)+C M(B+Z)}{A+B+Z}\right)}{1-(1-\eta)\left(\frac{-E(A+B+Z)+C M(B+Z)}{A+B+Z}\right)} .
$$

Theorem 3.1 If $R_{0}<1$, then system (3) is stable at $E^{0}$.

Proof Since $A, B$, and $C$ are positive and $\eta \in(0,1)$, we easily see that $\lambda_{2}<0$. By the assumption we have $R_{0}=\frac{C M(B+Z)}{E(A+B+Z)}<1$. Hence $C M(B+Z)-E(A+B+Z)<0$, and so $\eta\left(\frac{-E(A+B+Z)+C M(B+Z)}{A+B+Z}\right)<0$ and $1-(1-\eta)\left(\frac{-E(A+B+Z)+C M(B+Z)}{A+B+Z}\right)>0$. This implies that $\lambda_{3}<0$. Thus system (3) is stable at $E^{0}$.

\section{Existence and uniqueness}

In this section, we prove that the system has a unique solution. For this purpose, we write system (3) as follows:

$$
\left\{\begin{array}{l}
{ }_{0}^{C F} D_{t}^{\eta} S(t)=G_{1}(t, S(t)), \\
{ }_{0}^{C F} D_{t}^{\eta} I(t)=G_{2}(t, I(t)), \\
{ }_{0}^{C F} D_{t}^{\eta} N(t)=G_{3}(t, N(t)) .
\end{array}\right.
$$


Using the integral operator introduced by Losada and Nieto [39] on both sides of the equations in the system, we obtain

$$
\left\{\begin{array}{l}
S(t)-S(0)=\frac{2(1-\eta)}{(2-\eta) M(\eta)} G_{1}(t, S(t))+\frac{2 \eta}{(2-\eta) M(\eta)} \int_{0}^{t} G_{1}(\eta, S) d \eta, \\
I(t)-I(0)=\frac{2(1-\eta)}{(2-\eta) M(\eta)} G_{2}(t, I(t))+\frac{2 \eta}{(2-\eta) M(\eta)} \int_{0}^{t} G_{2}(\eta, I) d \eta, \\
N(t)-N(0)=\frac{2(1-\eta)}{(2-\eta) M(\eta)} G_{3}(t, N(t))+\frac{2 \eta}{(2-\eta) M(\eta)} \int_{0}^{t} G_{3}(\eta, N) d \eta .
\end{array}\right.
$$

First, we show that the kernels $G_{1}, G_{2}, G_{3}$ satisfy the Lipschitz condition and are contractions.

Theorem 4.1 The kernel $G_{1}$ satisfies the Lipschitz condition and is a contraction if

$$
0 \leq\left(-Z-D-A-C m_{1}\right)<1
$$

Proof For $S$ and $S_{1}$, we have

$$
\begin{aligned}
& \left\|G_{1}(t, S)-G_{1}\left(t, S_{1}\right)\right\| \\
& \quad=\left\|-Z\left(S(t)-S_{1}(t)\right)-D\left(S(t)-S_{1}(t)\right)-A\left(S(t)-S_{1}(t)\right)-C I(t)\left(S(t)-S_{1}(t)\right)\right\| \\
& \quad \leq(-Z-D-A-C\|I(t)\|)\left\|S(t)-S_{1}(t)\right\| \leq b_{1}\left\|S(t)-S_{1}(t)\right\| .
\end{aligned}
$$

Let $b_{1}=-Z-D-A-C\|I(t)\|$, where $I$ is a bounded function: $\|I(t)\| \leq m_{1}$. So

$$
\left\|G_{1}(t, S)-G_{1}\left(t, S_{1}\right)\right\| \leq b_{1}\left\|S(t)-S_{1}(t)\right\| .
$$

So $G_{1}$ satisfies the Lipschitz condition, and if $0 \leq(-Z-D-A-C\|I(t)\|)<1$, then $G_{1}$ is a contraction.

Similarly, $G_{2}$ and $G_{3}$ satisfy the Lipschitz conditions:

$$
\left\{\begin{array}{l}
\left\|G_{2}(t, I)-G_{2}\left(t, I_{1}\right)\right\| \leq b_{2}\left\|\left(I(t)-I_{1}(t)\right)\right\|, \\
\left\|G_{3}(t, N)-G_{3}\left(t, N_{1}\right)\right\| \leq b_{3}\left\|\left(N(t)-N_{1}(t)\right)\right\|,
\end{array}\right.
$$

where $b_{2}=B-D-E+C\|S(t)\|$ and $b_{3}=B-D$, and $S$ is a bounded function $\left(\|S(t)\| \leq m_{2}\right)$. If $0 \leq(B-D-E+C\|S\|)<1$ and $0 \leq B-D<1$, then $G_{2}$ and $G_{3}$ are contractions.

According to system (7), consider the following recursive forms:

$$
\begin{aligned}
H_{1 n}(t)= & S_{n}(t)-S_{n-1}(t) \\
= & \frac{2(1-\eta)}{(2-\eta) M(\eta)}\left(G_{1}\left(t, S_{n-1}\right)-G_{1}\left(t, S_{n-2}\right)\right) \\
& +\frac{2 \eta}{(2-\eta) M(\eta)} \int_{0}^{t}\left(G_{1}\left(\eta, S_{n-1}\right)-G_{1}\left(\eta, S_{n-2}\right)\right) d \eta, \\
H_{2 n}(t)= & I_{n}(t)-I_{n-1}(t) \\
= & \frac{2(1-\eta)}{(2-\eta) M(\eta)}\left(G_{2}\left(t, I_{n-1}\right)-G_{2}\left(t, I_{n-2}\right)\right) \\
& +\frac{2 \eta}{(2-\eta) M(\eta)} \int_{0}^{t}\left(G_{2}\left(\eta, I_{n-1}\right)-G_{2}\left(\eta, I_{n-2}\right)\right) d \eta,
\end{aligned}
$$




$$
\begin{aligned}
H_{3 n}(t)= & N_{n}(t)-N_{n-1}(t) \\
= & \frac{2(1-\eta)}{(2-\eta) M(\eta)}\left(G_{3}\left(t, N_{n-1}\right)-G_{3}\left(t, N_{n-2}\right)\right) \\
& +\frac{2 \eta}{(2-\eta) M(\eta)} \int_{0}^{t}\left(G_{3}\left(\eta, N_{n-1}\right)-G_{3}\left(\eta, N_{n-2}\right)\right) d \eta
\end{aligned}
$$

with initial conditions $S_{0}(t)=S(0), I_{0}(t)=I(0)$, and $N_{0}(t)=N(0)$. We take the norm of the first equation in the system:

$$
\begin{aligned}
\left\|H_{1 n}(t)\right\|= & \left\|S_{n}(t)-S_{n-1}(t)\right\| \\
= & \| \frac{2(1-\eta)}{(2-\eta) M(\eta)}\left(G_{1}\left(t, S_{n-1}\right)-G_{1}\left(t, S_{n-2}\right)\right) \\
& +\frac{2 \eta}{(2-\eta) M(\eta)} \int_{0}^{t}\left(G_{1}\left(\eta, S_{n-1}\right)-G_{1}\left(\eta, S_{n-2}\right)\right) d \eta \| \\
\leq & \frac{2(1-\eta)}{(2-\eta) M(\eta)}\left\|G_{1}\left(t, S_{n-1}\right)-G_{1}\left(t, S_{n-2}\right)\right\| \\
& +\frac{2 \eta}{(2-\eta) M(\eta)}\left\|\int_{0}^{t}\left(G_{1}\left(\eta, S_{n-1}\right)-G_{1}\left(\eta, S_{n-2}\right)\right) d \eta\right\| .
\end{aligned}
$$

With Lipschitz condition (8), we have

$$
\left\|H_{1 n}(t)\right\| \leq \frac{2(1-\eta)}{(2-\eta) M(\eta)} b_{1}\left\|H_{1(n-1)}(t)\right\|+\frac{2 \eta}{(2-\eta) M(\eta)} b_{1} \int_{0}^{t}\left\|H_{1(n-1)}(\eta)\right\| d \eta .
$$

Similarly, we obtain

$$
\begin{aligned}
\left\|H_{2 n}(t)\right\| & \leq \frac{2(1-\eta)}{(2-\eta) M(\eta)} b_{2}\left\|H_{2(n-1)}(t)\right\|+\frac{2 \eta}{(2-\eta) M(\eta)} b_{2} \int_{0}^{t}\left\|H_{2(n-1)}(\eta)\right\| d \eta, \\
\left\|H_{3 n}(t)\right\| & \leq \frac{2(1-\eta)}{(2-\eta) M(\eta)} b_{3}\left\|H_{1(n-1)}(t)\right\|+\frac{2 \eta}{(2-\eta) M(\eta)} b_{3} \int_{0}^{t}\left\|H_{3(n-1)}(\eta)\right\| d \eta .
\end{aligned}
$$

Then we can write that

$$
S_{n}(t)=\sum_{i=1}^{n} H_{1 i}(t), I_{n}(t)=\sum_{i=1}^{n} H_{2 i}(t), N_{n}(t)=\sum_{i=1}^{n} H_{3 i}(t) .
$$

To prove the existence of a solution, we state the following theorem.

Theorem 4.2 The Rabies Caputo-Fabrizio fractional model (3) has a solution if there exists $t_{1}$ such that

$$
\frac{2(1-\eta)}{(2-\eta) M(\eta)} b_{i}+\frac{2 \eta t_{1}}{(2-\eta) M(\eta)} b_{i}<1
$$


Proof From recursive technique, by Eqs. (9) and (10) we conclude that

$$
\begin{aligned}
\left\|H_{1 n}(t)\right\| & \leq\left\|S_{n}(0)\right\|\left[\frac{2(1-\eta)}{(2-\eta) M(\eta)} b_{1}+\frac{2 \eta t_{1}}{(2-\eta) M(\eta)} b_{1} t\right]^{n}, \\
\left\|H_{2 n}(t)\right\| & \leq\left\|I_{n}(0)\right\|\left[\frac{2(1-\eta)}{(2-\eta) M(\eta)} b_{2}+\frac{2 \eta t_{1}}{(2-\eta) M(\eta)} b_{2} t\right]^{n}, \\
\left\|H_{3 n}(t)\right\| & \leq\left\|N_{n}(0)\right\|\left[\frac{2(1-\eta)}{(2-\eta) M(\eta)} b_{3}+\frac{2 \eta t_{1}}{(2-\eta) M(\eta)} b_{3} t\right]^{n} .
\end{aligned}
$$

Then the system has a solution, and also it is continuous. Now we show that the above functions give a solution for model (3). Let

$$
\begin{aligned}
& S(t)-S(0)=S_{n}(t)-\mathrm{B}_{1 n}(t), \\
& I(t)-I(0)=I_{n}(t)-\mathrm{B}_{2 n}(t), \\
& N(t)-N(0)=N_{n}(t)-\mathrm{B}_{3 n}(t) .
\end{aligned}
$$

Hence

$$
\begin{aligned}
\left\|\mathrm{B}_{1 n}(t)\right\|= & \| \frac{2(1-\eta)}{(2-\eta) M(\eta)}\left(G_{1}(t, S)-G_{1}\left(t, S_{n-1}\right)\right) \\
& +\frac{2 \eta}{(2-\eta) M(\eta)} \int_{0}^{t}\left(G_{1}(\eta, S)-G_{1}\left(\eta, S_{n-1}\right)\right) d \eta \| \\
\leq & \frac{2(1-\eta)}{(2-\eta) M(\eta)}\left\|G_{1}(t, S)-G_{1}\left(t, S_{n-1}\right)\right\| \\
& +\frac{2 \eta}{(2-\eta) M(\eta)} \int_{0}^{t}\left\|G_{1}(\eta, S)-G_{1}\left(\eta, S_{n-1}\right)\right\| d \eta \\
\leq & \frac{2(1-\eta)}{(2-\eta) M(\eta)} b_{1}\left\|S-S_{n-1}\right\|+\frac{2 \eta}{(2-\eta) M(\eta)} b_{1}\left\|S-S_{n-1}\right\| t .
\end{aligned}
$$

By repeating the method we obtain

$$
\left\|\mathrm{B}_{1 n}(t)\right\| \leq\left[\frac{2(1-\eta)}{(2-\eta) M(\eta)}+\frac{2 \eta}{(2-\eta) M(\eta)} t\right]^{n+1} b_{1}^{n+1} h .
$$

At $t_{1}$, we get

$$
\left\|\mathrm{B}_{1 n}(t)\right\| \leq\left[\frac{2(1-\eta)}{(2-\eta) M(\eta)}+\frac{2 \eta}{(2-\eta) M(\eta)} t_{1}\right]^{n+1} b_{1}^{n+1} h
$$

Taking the limit as $n$ tends to $\infty$, we obtain $\left\|\mathrm{B}_{1 n}(t)\right\| \rightarrow 0$. Similarly, we can show that $\left\|\mathrm{B}_{2 n}(t)\right\| \rightarrow 0$ and $\left\|\mathrm{B}_{3 n}(t)\right\| \rightarrow 0$, and the proof is complete. 
To show the uniqueness of a solution, suppose that the system has another solution $S_{1}(t)$, $I_{1}(t)$, and $N_{1}(t)$. Then we have

$$
\begin{aligned}
S(t)-S_{1}(t)= & \frac{2(1-\eta)}{(2-\eta) M(\eta)}\left(G_{1}(t, S)-G_{1}\left(t, S_{1}\right)\right) \\
& +\frac{2 \eta}{(2-\eta) M(\eta)} \int_{0}^{t}\left(G_{1}(\eta, S)-G_{1}\left(\eta, S_{1}\right)\right) d \eta .
\end{aligned}
$$

Taking the norm, we have

$$
\begin{aligned}
\left\|S(t)-S_{1}(t)\right\| \leq & \frac{2(1-\eta)}{(2-\eta) M(\eta)}\left\|G_{1}(t, S)-G_{1}\left(t, S_{1}\right)\right\| \\
& +\frac{2 \eta}{(2-\eta) M(\eta)} \int_{0}^{t}\left\|G_{1}(\eta, S)-G_{1}\left(\eta, S_{1}\right)\right\| d \eta .
\end{aligned}
$$

From Lipschitz condition (8) it follows that

$$
\left\|S(t)-S_{1}(t)\right\| \leq \frac{2(1-\eta)}{(2-\eta) M(\eta)} b_{1}\left\|S(t)-S_{1}(t)\right\|+\frac{2 \eta}{(2-\eta) M(\eta)} b_{1} t\left\|S(t)-S_{1}(t)\right\| .
$$

So

$$
\left\|S(t)-S_{1}(t)\right\|\left(1-\frac{2(1-\eta)}{(2-\eta) M(\eta)} b_{1}-\frac{2 \eta}{(2-\eta) M(\eta)} b_{1} t\right) \leq 0 .
$$

Theorem 4.3 The solution of the Rabies model is unique if

$$
1-\frac{2(1-\eta)}{(2-\eta) M(\eta)} b_{1}-\frac{2 \eta}{(2-\eta) M(\eta)} b_{1} t>0 .
$$

Proof Suppose that condition (11) holds. Then $\left\|S(t)-S_{1}(t)\right\|=0$, and so $S(t)=S_{1}(t)$. Similarly, we can show the same equality for $I$ and $N$.

\section{Solution of equations by Laplace Adomian decomposition method}

In this section, we solve the fractional-order model of Rabies by the Laplace Adomian decomposition method. Applying the Laplace transform to both sides of system (3), we have

$$
\left\{\begin{array}{l}
L\left\{\left\{_{0}^{C F} D_{t}^{\eta} S(t)\right\}=L\{B N(t)+Z(N(t)-S(t)-I(t))-D S(t)-A S(t)-B I(t)-C S(t) I(t)\}\right. \\
L\left\{{ }_{0}^{C F} D_{t}^{\eta} I(t)\right\}=L\{B I(t)+C S(t) I(t)-D I(t)-E I(t)\} \\
L\left\{{ }_{0}^{C F} D_{t}^{\eta} N(t)\right\}=L\{N(t)(B-D)-E I(t)\}
\end{array}\right.
$$

and so

$$
\left\{\begin{array}{l}
\frac{s L(S)-S(0)}{s+\eta(1-s)}=L[B N(t)+Z(N(t)-S(t)-I(t))-D S(t)-A S(t)-B I(t)-C S(t) I(t)], \\
\frac{s L(I)-I(0)}{s+\eta(1-s)}=L[B I(t)+C S(t) I(t)-D I(t)-E I(t)], \\
\frac{s L(N)-N(0)}{s+\eta(1-s)}=L[N(t)(B-D)-E I(t)] .
\end{array}\right.
$$


Using the initial conditions and taking the inverse Laplace transform of these equations, we obtain

$$
\left\{\begin{aligned}
S(t)= & S(0)+L^{-1}\left[\frac{s+\eta(1-s)}{s} L\{B N(t)+Z(N(t)-S(t)-I(t))\right. \\
& -D S(t)-A S(t)-B I(t)-C S(t) I(t)\}] \\
I(t)= & I(0)+L^{-1}\left[\frac{s+\eta(1-s)}{s} L\{B I(t)+C S(t) I(t)-D I(t)-E I(t)\}\right] \\
N(t)= & N(0)+L^{-1}\left[\frac{s+\eta(1-s)}{s} L\{N(t)(B-D)-E I(t)\}\right] .
\end{aligned}\right.
$$

Suppose that the solutions $S, I, N$ can be written in the form of infinite series

$$
S(t)=\sum_{n=0}^{\infty} S_{n}(t), \quad I(t)=\sum_{n=0}^{\infty} I_{n}(t), \quad N(t)=\sum_{n=0}^{\infty} N_{n}(t)
$$

and that the nonlinear term SI involved in the model can be decomposed by Adomian polynomials as

$$
S(t) I(t)=\sum_{n=0}^{\infty} P_{n}(t)
$$

Recall that the Adomian polynomials $P_{n}$ are defined as

$$
P_{n}(t)=\left.\frac{1}{\Gamma(n+1)} \frac{d^{n}}{d \lambda^{n}}\left[\sum_{k=0}^{n} \lambda^{k} S_{k}(t) \sum_{k=0}^{n} \lambda^{k} I_{k}(t)\right]\right|_{\lambda=0} .
$$

The first three polynomials are given by

$$
\left\{\begin{array}{l}
P_{0}(t)=S_{0}(t) I_{0}(t), \\
P_{1}(t)=S_{0}(t) I_{1}(t)+S_{1}(t) I_{0}(t), \\
P_{2}(t)=2 S_{0}(t) I_{2}(t)+2 S_{1}(t) I_{1}(t)+2 S_{2}(t) I_{0}(t) .
\end{array}\right.
$$

Using (13), (14), and (15) in model (12), we get

$$
\begin{aligned}
L\left\{\sum_{n=0}^{\infty} S_{n}(t)\right\}= & \frac{S(0)}{s}+\left[\frac { s + \eta ( 1 - s ) } { s } L \left\{B \sum_{n=0}^{\infty} N_{n}(t)\right.\right. \\
& +Z\left(\sum_{n=0}^{\infty} N_{n}(t)-\sum_{n=0}^{\infty} S_{n}(t)-\sum_{n=0}^{\infty} I_{n}(t)\right) \\
& \left.\left.-(D+A) \sum_{n=0}^{\infty} S_{n}(t)-B \sum_{n=0}^{\infty} I_{n}(t)-C \sum_{n=0}^{\infty} P_{n}(t)\right\}\right], \\
L\left\{\sum_{n=0}^{\infty} I_{n}(t)\right\}= & \frac{I(0)}{s}+\left[\frac{s+\eta(1-s)}{s} L\left\{B \sum_{n=0}^{\infty} I_{n}(t)+C \sum_{n=0}^{\infty} P_{n}(t)-(D+E) \sum_{n=0}^{\infty} I_{n}(t)\right\}\right] \\
L\left\{\sum_{n=0}^{\infty} N_{n}(t)\right\}= & \frac{N(0)}{s}+\left[\frac{s+\eta(1-s)}{s} L\left\{(B-D) \sum_{n=0}^{\infty} N_{n}(t)-E \sum_{n=0}^{\infty} I_{n}(t)\right\}\right] .
\end{aligned}
$$


By matching the two sides of equations we obtain

$$
\begin{aligned}
& \left\{\begin{aligned}
L\left(S_{0}\right)= & \frac{S_{0}}{s}, \\
L\left(S_{1}\right)= & \frac{s+\eta(1-s)}{s}\left[B L\left\{N_{0}\right\}+Z\left(L\left\{N_{0}\right\}-L\left\{S_{0}\right\}-L\left\{I_{0}\right\}\right)\right. \\
& \left.-(D+A) L\left\{S_{0}\right\}-B L\left\{I_{0}\right\}-C L\left\{P_{0}\right\}\right], \\
L\left(S_{2}\right)= & \frac{s+\eta(1-s)}{s}\left[B L\left\{N_{1}\right\}+Z\left(L\left\{N_{1}\right\}-L\left\{S_{1}\right\}-L\left\{I_{1}\right\}\right)\right. \\
& \left.-(D+A) L\left\{S_{1}\right\}-B L\left\{I_{1}\right\}-C L\left\{P_{1}\right\}\right], \\
\vdots & \left.\quad-(D+A) L\left\{S_{n}\right\}-B L\left\{I_{n}\right\}-C L\left\{P_{n}\right\}\right] ;
\end{aligned}\right. \\
& \left\{\begin{array}{l}
L\left(I_{0}\right)=\frac{I_{0}}{s}, \\
L\left(I_{1}\right)=\frac{s+\eta(1-s)}{s}\left[B L\left\{I_{0}\right\}+C L\left\{P_{0}\right\}-D L\left\{I_{0}\right\}-E L\left\{I_{0}\right\}\right], \\
L\left(I_{2}\right)=\frac{s+\eta(1-s)}{s}\left[B L\left\{I_{1}\right\}+C L\left\{P_{1}\right\}-D L\left\{I_{1}\right\}-E L\left\{I_{1}\right\}\right], \\
\vdots \\
L\left(I_{n+1}\right)=\frac{s+\eta(1-s)}{s}\left[B L\left\{I_{n}\right\}+C L\left\{P_{n}\right\}-D L\left\{I_{n}\right\}-E L\left\{I_{n}\right\}\right] ;
\end{array}\right. \\
& \left\{\begin{array}{l}
L\left(N_{0}\right)=\frac{N_{0}}{s}, \\
L\left(N_{1}\right)=\frac{s+\eta(1-s)}{s}\left[(B-D) L\left\{N_{0}\right\}-E L\left\{I_{0}\right\}\right], \\
L\left(N_{2}\right)=\frac{s+\eta(1-s)}{s}\left[(B-D) L\left\{N_{1}\right\}-E L\left\{I_{1}\right\}\right], \\
\vdots \\
L\left(N_{n+1}\right)=\frac{s+\eta(1-s)}{s}\left[(B-D) L\left\{N_{n}\right\}-E L\left\{I_{n}\right\}\right] .
\end{array}\right.
\end{aligned}
$$

Taking the Laplace inverse on both sides of the previous equations, we have

$$
\left\{\begin{aligned}
S_{0}= & S_{0}, \quad I_{0}=I_{0}, \quad N_{0}=N_{0}, \\
S_{1}= & {\left[B N_{0}+Z\left(N_{0}-I_{0}-S_{0}\right)-D S_{0}-A S_{0}-B I_{0}-C I_{0} S_{0}\right](1+\eta(t-1)), } \\
I_{1}= & {\left[B I_{0}+C I_{0} S_{0}-D I_{0}-E I_{0}\right](1+\eta(t-1)), } \\
N_{1}= & {\left[(B-D) N_{0}-E I_{0}\right](1+\eta(t-1)), S_{2}=\left[(B+Z)\left((B-D) N_{0}-E I_{0}\right)\right.} \\
& -\left(Z+D+A+C I_{0}\right)\left((B+Z) N_{0}-(Z+D+A) S_{0}+\left(-Z-B-C S_{0}\right) I_{0}\right) \\
& \left.+\left(-Z-B-C S_{0}\right)\left(B+C S_{0}-D-E\right) I_{0}\right]\left(\frac{1}{2} \eta^{2} t^{2}-2 t \eta^{2}+2 \eta t+(\eta-1)^{2}\right), \\
I_{2}= & {\left[\left(B-D-E+C S_{0}\right)^{2} I_{0}+C I_{0}\left((B+Z) N_{0}\right.\right.} \\
& \left.\left.-(Z+D+A) S_{0}+\left(Z-B-C S_{0}\right) I_{0}\right)\right]\left(\frac{1}{2} \eta^{2} t^{2}-2 t \eta^{2}+2 \eta t+(\eta-1)^{2}\right), \\
N_{2}= & {\left[(B-D)^{2} N_{0}-E I_{0}\left(2 B-2 D+C S_{0}-E\right)\right]\left(\frac{1}{2} \eta^{2} t^{2}-2 t \eta^{2}+2 \eta t+(\eta-1)^{2}\right) . }
\end{aligned}\right.
$$

Similarly, we can obtain the remaining terms. Finally, we get the solution in the form of infinite series given by

$$
S(t)=\sum_{n=0}^{\infty} S_{n}, \quad I(t)=\sum_{n=0}^{\infty} I_{n}, \quad N(t)=\sum_{n=0}^{\infty} N_{n} .
$$




\subsection{Convergence analysis}

The series obtained from the Adomian method converge rapidly and uniformly to the exact solution of the system. For this purpose, we use the classical method described in [38]. The series of solutions obtained by the Adomian decomposition method (16) can be described as follows (see [47]):

$$
u_{n}=T u_{n-1}, \quad u_{n-1}=\sum_{i=1}^{n} u_{i}, \quad n=1,2,3, \ldots
$$

We state the convergence conditions of $\left\{u_{n}\right\}$ in the following theorem $[38,48,49]$.

Theorem 5.1 Let $\Omega$ be a Banach space, and let $T: \Omega \rightarrow \Omega$ be a contraction map with contractive constant $0<h<1$, Then $T$ has a unique point $u$ such that $T(u)=u$, where $u=$ $(S, I, N)$. Let $u_{0}=u_{0} \in F_{r}(u)$, where $F_{r}(u)=\left\{u^{\prime} \in \Omega:\left\|u^{\prime}-u\right\|<r\right\}$. Then we have $u_{n} \in F_{r}(u)$ for all $n$ and $u_{n} \rightarrow u$.

Proof We use mathematical induction. For $n=1$, we have

$$
\left\|u_{0}-u\right\|=\left\|T\left(u_{0}\right)-T(u)\right\| \leq h\left\|u_{0}-x\right\| .
$$

If the statement is true for $n-1$, then $\left\|u_{n-1}-u\right\| \leq h^{n-1}\left\|u_{0}-u\right\|$. So

$$
\left\|u_{n}-u\right\|=\left\|T\left(u_{n-1}\right)-T(u)\right\| \leq h\left\|u_{n-1}-u\right\| \leq h^{n}\left\|u_{0}-u\right\| .
$$

Also, $u_{0} \in F_{r}(u)$, and consequently $\left\|u_{0}-u\right\|<r$, so we get $\left\|u_{n}-u\right\| \leq h^{n}\left\|u_{0}-u\right\| \leq h^{n} r<r$. This implies that $u_{n} \in F_{r}(u)$. Furthermore, since $\left\|u_{n}-u\right\| \leq h^{n}\left\|u_{0}-u\right\|$ and $\lim _{n \rightarrow \infty} h^{n}=0$, we have $\lim _{n \rightarrow \infty}\left\|u_{n}-u\right\|=0$, and then $\lim _{n \rightarrow \infty} u_{n}=u$, and the proof is complete.

\section{Stability of solution}

In this section, we first introduce a special solution to the Rabies model by using the Sumudu transform and then prove the stability of the method by a fixed point theorem.

\subsection{Special solution by using Sumudu transform}

Here we provide a special solution to the Rabies model. Applying the Sumudu transform to system (3), we get

$$
\left\{\begin{aligned}
\left.S T{ }_{0}^{C F} D_{t}^{\eta} S(t)\right)= & S T[B N(t)+Z(N(t)-S(t) \\
& -I(t))-D S(t)-A S(t)-B I(t)-C S(t) I(t)], \\
S T\left({ }_{0}^{C F} D_{t}^{\eta} I(t)\right)= & S T[B I(t)+C S(t) I(t)-D I(t)-E I(t)] \\
S T\left({ }_{0}^{C F} D_{t}^{\eta} N(t)\right)= & S T[N(t)(B-D)-E I(t)] .
\end{aligned}\right.
$$

By the definition of the Sumudu transform of CF-derivative we obtain

$$
\left\{\begin{aligned}
\frac{M(\eta)}{1-\eta+\eta u}(S T(S(t))-S(0))= & S T[B N(t)+Z(N(t) \\
& -S(t)-I(t))-D S(t)-A S(t)-B I(t)-C S(t) I(t)], \\
\frac{M(\eta)}{1-\eta+\eta u}(S T(I(t))-I(0))= & S T[B I(t)+C S(t) I(t)-D I(t)-E I(t)], \\
\frac{M(\eta)}{1-\eta+\eta u}(S T(N(t))-N(0))= & S T[N(t)(B-D)-E I(t)] .
\end{aligned}\right.
$$


Rearranging, we obtain following equalities:

$$
\left\{\begin{aligned}
S T(S(t))= & S(0)+\frac{1-\eta+\eta u}{M(\eta)} S T[B N(t)+Z(N(t)-S(t) \\
& -I(t))-D S(t)-A S(t)-B I(t)-C S(t) I(t)] \\
S T(I(t))= & I(0)+\frac{1-\eta+\eta u}{M(\eta)} S T[B I(t)+C S(t) I(t)-D I(t)-E I(t)] \\
S T(N(t))= & N(0)+\frac{1-\eta+\eta u}{M(\eta)} S T[N(t)(B-D)-E I(t)]
\end{aligned}\right.
$$

We obtain the following recursive formulas:

$$
\left\{\begin{aligned}
S_{n+1}(t)= & S_{n}(0)+S T^{-1}\left\{\frac{1-\eta+\eta u}{M(\eta)} S T[B N(t)+Z(N(t)-S(t)\right. \\
& -I(t))-D S(t)-A S(t)-B I(t)-C S(t) I(t)]\} \\
I_{n+1}(t)= & I_{n}(0)+S T^{-1}\left\{\frac{1-\eta+\eta u}{M(\eta)} S T[B I(t)+C S(t) I(t)-D I(t)-E I(t)]\right\} \\
N_{n+1}(t)= & N_{n}(0)+\frac{1-\eta+\eta u}{M(\eta)} S T[N(t)(B-D)-E I(t)] .
\end{aligned}\right.
$$

Finally, the solution of system (3) is approximates as follows:

$$
S(t)=\lim _{n \rightarrow \infty} S_{n}(t), \quad I(t)=\lim _{n \rightarrow \infty} I_{n}(t), \quad N(t)=\lim _{n \rightarrow \infty} N_{n}(t) .
$$

\subsection{Stability analysis of iteration method}

Consider a Banach space $(Y,\|\cdot\|)$, a self-map $F$ on $Y$, and a recursive method $P_{n+1}=$ $\phi\left(F, P_{n}\right)$. Denote by $\Omega(F) \neq \emptyset$ the fixed point set of $F$, and let $\lim _{n \rightarrow \infty} P_{n}=p \in \Omega(F)$. Let $\left\{f_{n}\right\} \subset \Omega$ and $e_{n}=\left\|f_{n+1}-\phi\left(F, f_{n}\right)\right\|$. If $\lim _{n \rightarrow \infty} e_{n}=0$ implies that $\lim _{n \rightarrow \infty} f_{n}=p$, then the recursive procedure $P_{n+1}=\phi\left(F, P_{n}\right)$ is $F$-stable. Suppose that our sequence $\left\{f_{n}\right\}$ has an upper bound. If Picard's iteration $P_{n+1}=F P_{n}$ satisfies all these conditions, then it is $F$-stable.

Theorem 6.1 ([44]) Let $(Y,\|\cdot\|)$ be a Banach space, and let $F$ be a self-map of $Y$ satisfying

$$
\left\|F_{x}-F_{y}\right\| \leq H\left\|x-F_{x}\right\|+h\|x-y\|
$$

for all $x, y \in Y$ where $H \geq 0$ and $0 \leq h<1$. Suppose that $F$ is Picard F-stable.

Suppose that the fractional model of Rabies (3) is connected with the subsequent iterative formula (15). Consider the following theorem.

Theorem 6.2 Let $F$ be a self-map defined as follows:

$$
\left\{\begin{aligned}
F\left(S_{n}(t)\right)= & S_{n+1}(t) \\
= & S_{n}(t)+S T^{-1}\left\{\frac{1-\eta+\eta u}{M(\eta)} S T[B N(t)+Z(N(t)\right. \\
& -S(t)-I(t))-D S(t)-A S(t)-B I(t)-C S(t) I(t)]]\}, \\
F\left(I_{n}(t)\right)= & I_{n+1}(t) \\
= & I_{n}(t)+S T^{-1}\left\{\frac{1-\eta+\eta u}{M(\eta)} S T[B I(t)+C S(t) I(t)-D I(t)-E I(t)]\right\}, \\
F\left(N_{n}(t)\right)= & N_{n+1}(t)=N_{n}(t)+\frac{1-\eta+\eta u}{M(\eta)} S T[N(t)(B-D)-E I(t)] .
\end{aligned}\right.
$$


This iterative recursion is $F$-stable in $L^{1}(a, b)$ if the following conditions are satisfied:

$$
\left\{\begin{array}{l}
\left(1+(B+Z) f_{1}(v)+(Z+D+A) f_{2}(v)+(Z+B) f_{3}(v)+C L_{2} f_{4}(v)+C L_{1} f_{5}(v)\right)<1 \\
\left(1+(B-D-E) f_{6}(v)+C L_{2} f_{7}(v)+C L_{1} f_{8}(v)\right)<1, \\
\left(1+(B-D) f_{9}(v)-E f_{10}(v)\right)<1 .
\end{array}\right.
$$

Proof First, we compute the following equality for $(n, m) \in N \times N$ to prove that $F$ has a fixed point:

$$
\begin{aligned}
F\left(S_{n}(t)\right. & -F\left(S_{m}(t)\right) \\
= & S_{n}(t)-S_{m}(t) \\
& +S T^{-1}\left\{\frac{1-\eta+\eta u}{M(\eta)} S T\left[(B+Z) N_{n}+(-Z-D-A) S_{n}+(-Z-B) I_{n}-C S_{n} I_{n}\right]\right. \\
& -S T^{-1}\left\{\frac{1-\eta+\eta u}{M(\eta)} S T\left[(B+Z) N_{m}+(-Z-D-A) S_{m}+(-Z-B) I_{m}-C S_{m} I_{m}\right]\right\} .
\end{aligned}
$$

Applying the norm on both sides, we obtain

$$
\begin{aligned}
\| F( & \left.S_{n}(t)\right)-F\left(S_{m}(t)\right) \\
= & \| S_{n}(t)-S_{m}(t)+S T^{-1}\left\{\frac { 1 - \eta + \eta u } { M ( \eta ) } S T \left[(B+Z) N_{n}+(-Z-D-A) S_{n}+(-Z-B) I_{n}\right.\right. \\
& \left.\quad-C S_{n} I_{n}\right]-S T^{-1}\left\{\frac { 1 - \eta + \eta u } { M ( \eta ) } S T \left[(B+Z) N_{m}\right.\right. \\
& \left.\left.+(-Z-D-A) S_{m}+(-Z-B) I_{m}-C S_{m} I_{m}\right]\right\} \| \\
\leq & \left\|S_{n}(t)-S_{m}(t)\right\|+S T^{-1}\left\{\frac { 1 - \eta + \eta u } { M ( \eta ) } S T \left[\left\|(B+Z)\left(N_{n}-N_{m}\right)\right\|\right.\right. \\
& +\left\|(-Z-D-A)\left(S_{n}-S_{m}\right)\right\| \\
& \left.+\left\|(-Z-B)\left(I_{n}-I_{m}\right)\right\|+\left\|-C I_{n}\left(S_{n}-S_{m}\right)\right\|+\left\|C S_{m}\left(I_{n}-I_{m}\right)\right\|\right]
\end{aligned}
$$

Because of the same role of both solutions, we consider

$$
\left\|S_{n}(t)-S_{m}(t)\right\| \cong\left\|I_{n}(t)-I_{m}(t)\right\| \cong\left\|N_{n}(t)-N_{m}(t)\right\| .
$$

From Eqs. (16) and (17) we obtain

$$
\begin{aligned}
& \| F\left(S_{n}(t)\right)-F\left(S_{m}(t)\right) \\
& \leq\left\|S_{n}(t)-S_{m}(t)\right\|+S T^{-1}\left\{\frac { 1 - \eta + \eta u } { M ( \eta ) } S T \left[(B+Z)\left\|\left(S_{n}-S_{m}\right)\right\|\right.\right. \\
& \quad+(Z+D+A)\left\|\left(S_{n}-S_{m}\right)\right\|+(Z+B)\left\|\left(S_{n}-S_{m}\right)\right\| \\
&\left.\quad+C\left\|I_{n}\right\|\left\|\left(S_{n}-S_{m}\right)\right\|+C\left\|S_{m}\right\|\left\|\left(S_{n}-S_{m}\right)\right\|\right] .
\end{aligned}
$$


Since $S_{n}, I_{n}, N_{n}$ are convergent sequences, they are bounded, so there exist $L_{1}, L_{2}, L_{3}$ for all $t$ such that

$$
\left\|S_{n}\right\|<L_{1}, \quad\left\|I_{n}\right\|<L_{2}, \quad\left\|N_{n}\right\|<L_{3}, \quad(m, n) \in N \times N .
$$

From Eqs. (18) and (19) we obtain

$$
\begin{aligned}
& \left\|F\left(S_{n}(t)\right)-F\left(S_{m}(t)\right)\right\| \\
& \leq \quad\left(1+(B+Z) f_{1}(v)+(Z+D+A) f_{2}(v)+(Z+B) f_{3}(v)\right. \\
& \left.\quad+C L_{2} f_{4}(v)+C L_{1} f_{5}(v)\right)\left\|S_{n}(t)-S_{m}(t)\right\|
\end{aligned}
$$

where $f_{i}$ are functions from $S T^{-1}\left[\frac{1-\eta+\eta u}{M(\eta)} S T[*]\right]$. In the same way, we get

$$
\begin{aligned}
& \| F\left(I_{n}(t)-F\left(I_{m}(t)\right) \|\right. \\
& \quad \leq\left(1+(B-D-E) f_{6}(v)+C L_{2} f_{7}(v)+C L_{1} f_{8}(v)\right)\left\|I_{n}(t)-I_{m}(t)\right\|, \\
& \| F\left(N_{n}(t)-F\left(N_{m}(t)\right)\left\|\leq\left(1+(B-D) f_{9}(v)-E f_{10}(v)\right)\right\| N_{n}(t)-N_{m}(t) \|,\right.
\end{aligned}
$$

where

$$
\left\{\begin{array}{l}
\left(1+(B+Z) f_{1}(v)+(Z+D+A) f_{2}(v)+(Z+B) f_{3}(v)+C L_{2} f_{4}(v)+C L_{1} f_{5}(v)\right)<1, \\
\left(1+(B-D-E) f_{6}(v)+C L_{2} f_{7}(v)+C L_{1} f_{8}(v)\right)<1, \\
\left(1+(B-D) f_{9}(v)-E f_{10}(v)\right)<1 .
\end{array}\right.
$$

Then the self-mapping $F$ has a fixed point. Let (20), (21), and (22) hold. We assume that

$$
\begin{aligned}
& H=(0,0,0), \\
& h=\left\{\begin{array}{l}
1+(B+Z) f_{1}(v)+(Z+D+A) f_{2}(v)+(Z+B) f_{3}(v)+C L_{2} f_{4}(v)+C L_{1} f_{5}(v), \\
1+(B-D-E) f_{6}(v)+C L_{2} f_{7}(v)+C L_{1} f_{8}(v), \\
1+(B-D) f_{9}(v)-E f_{10}(v) .
\end{array}\right.
\end{aligned}
$$

Then all conditions of Theorem 5.1 hold, and this completes the proof.

\section{Numerical results}

In this section, we give a numerical simulation of the results of solving the Rabies model (3) by using the Laplace Adomian decomposition method (LADM). This method provides the solution of the system in the form of infinite series. We have chosen the parameters $b=0.083, c=0.1, e=0.5, a=0, z=0, d=0.01+0.004 \mathrm{~N}$ and the initial conditions $S_{0}=9$, $I_{0}=1, N_{0}=10$ (see [5]). In Figs. 1 and 2, we show the solutions of model (4) for different values of $\eta=1,0.9,0.8,0.7,0.6,0.5$. The figures show that different fractional orders have an important effect on the dynamics of the system and indicate that as $\eta \rightarrow 1$, the approximate solutions tend to the classic integer solution with $\eta=1$. Also, figures show that every function has the same behavior for different values of $\eta$, but the obtained results for various values of $\eta$ are different. 

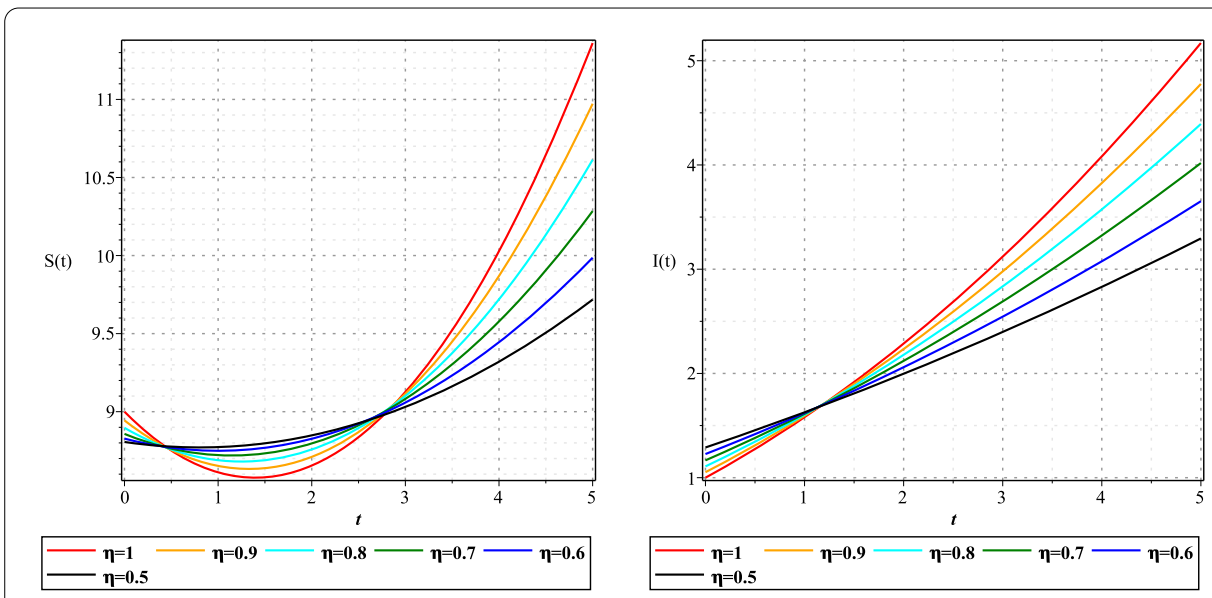

Figure 1 Dynamics of susceptible animals $S$ and infected animals / for various values of $\eta$

Figure 2 Dynamics of $N(t)$ (population of animals at time $t$ ) for various values of $\eta$

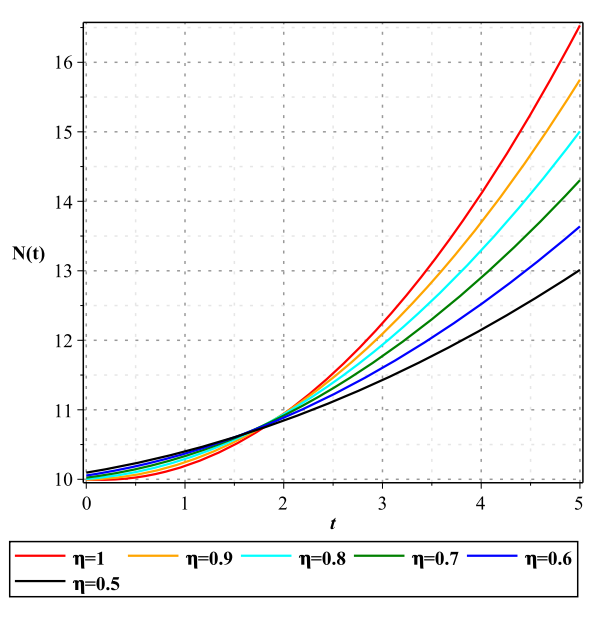

Figure 3 Dynamics of $/(t)$ for various values of $b$

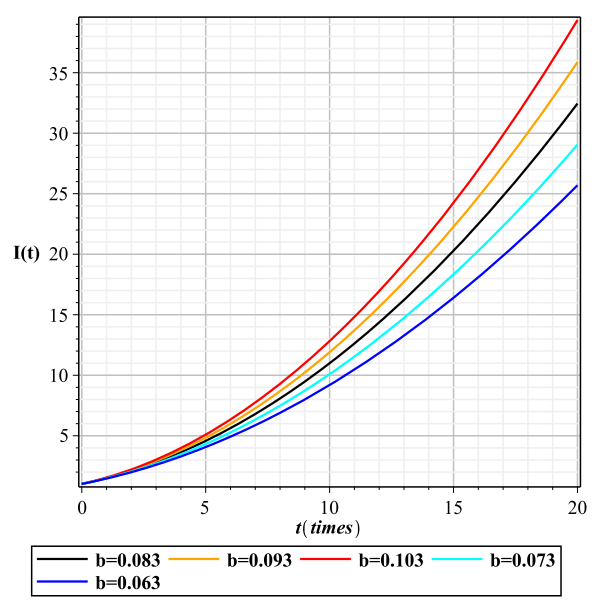

Figures 3-7 show the effect of coefficients on the number of infected animals $I(t)$. In Fig. 3, we plotted $I(t)$ for different values of $b$, and we see that increasing $b$ increases the 
Figure 4 Dynamics of $/(t)$ for various values of $z$

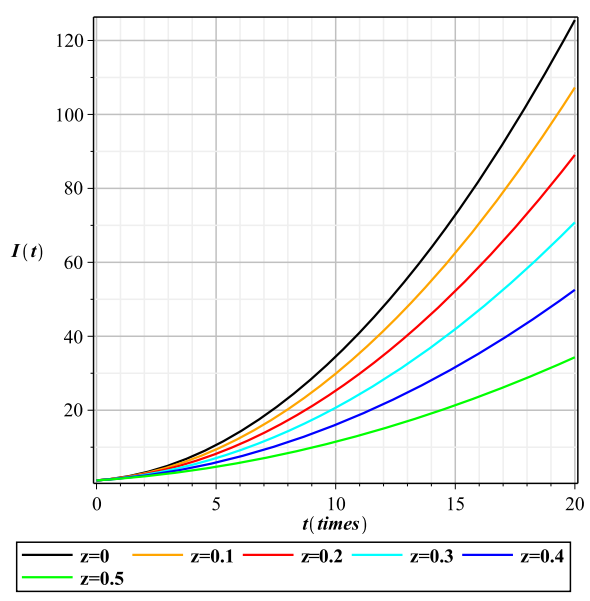

Figure 5 Dynamics of $/(t)$ for various values of $c$

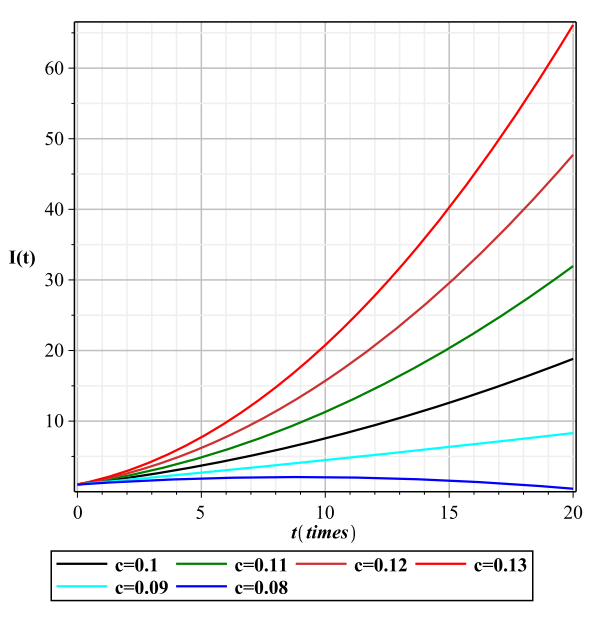

number of infected animals and decreasing $b$ reduces the number of infected animals; $I(t)$ for different values of $z$ is plotted in Fig. 4, and we see that increasing $z$ reduces the number of infected animals. Figure 5 shows the effect of Rabies transmission on the number of infected animals, and as w can see, $c$ has a direct effect on $I(t)$, and a slight increase or decrease causes a significant increase or decrease in the number of infected animals. Also, one way to control the disease is vaccination, which affects the number of infected animals as shown in Fig. 6 . With the increase in vaccination rate $a$, the number of infected animals rapidly decreases. The disease mortality rate also has a direct effect on the number of infected animals, and as Fig. 7 shows, increasing or decreasing $e$ causes a similar behavior of $I(t)$.

A comparison between the noninteger-order model with $\eta=0.98$ and the integer order $\eta=1$ is also given in Tables $1-3$. The results of all three types of derivatives for $S, I, N$ shown in Tables 1-3 indicate the similar behavior of functions in all three types of derivatives, but they are different in terms of values, and this difference is not uniform. At some points the value derived from the fractional-order Caputo derivative is closer to the result of integer order, and at other points the result of the fractional-order Caputo-Fabrizio derivative is closer to that of integer order. 
Figure 6 Dynamics of $/(t)$ for various values of $a$

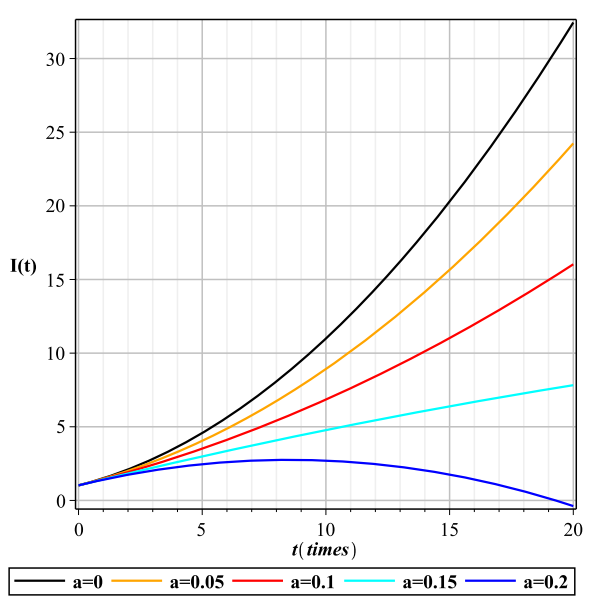

Figure 7 Dynamics of $/(t)$ for various values of $e$

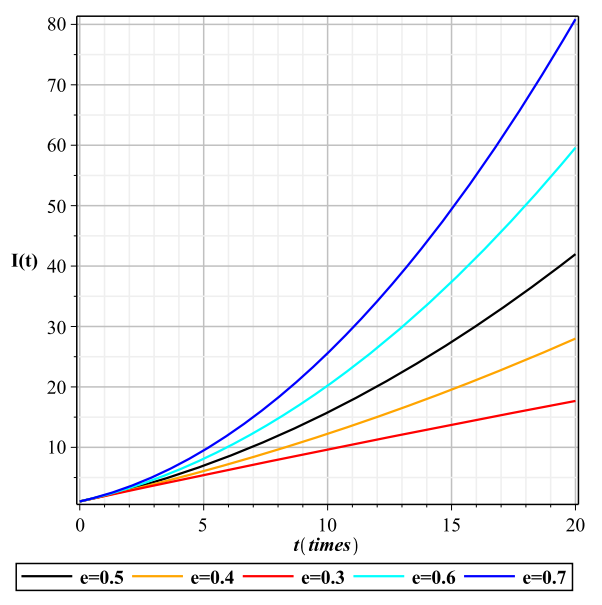

Table 1 Results of three types of derivative: Ordinary derivative $D^{v}$, Caputo fractional derivative ${ }^{c} D^{v}$, and Caputo-Fabrizio fractional derivative ${ }^{c f} D^{v}$ for $S(t)$

\begin{tabular}{lllllll}
\hline$t$ & 0 & 1 & 2 & 3 & 4 & 5 \\
\hline$D^{v}(v=1)$ & 9 & 8.61205 & 8.6542 & 9.12645 & 10.0288 & 11.36125 \\
${ }^{c} D^{v}(v=0.98)$ & 9 & 8.6150 & 8.6685 & 9.1367 & 10.011 & 11.285 \\
${ }^{c f} D^{v}(v=0.98)$ & 8.988112 & 8.620566 & 8.666090 & 9.124682 & 9.996344 & 11.281074 \\
\hline
\end{tabular}

Table 2 Results of three types of derivative: Ordinary derivative $D^{\nu}$, Caputo fractional derivative ${ }^{c} D^{\nu}$, and Caputo-Fabrizio fractional derivative ${ }^{c f} D^{v}$ for $/(t)$

\begin{tabular}{lllllll}
\hline$t$ & 0 & 1 & 2 & 3 & 4 & 5 \\
\hline$D^{v}(v=1)$ & 1 & 1.57959 & 2.28636 & 3.12031 & 4.08144 & 5.16975 \\
${ }^{c} D^{v}(v=0.98)$ & 1 & 1.586274 & 2.282928 & 3.0951379 & 4.0227778 & 5.065352 \\
${ }^{c f} D^{v}(v=0.98)$ & 1.010371 & 1.582113 & 2.276007 & 3.0920537 & 4.030253 & 5.090604 \\
\hline
\end{tabular}

\section{Conclusion}

In this work, we have studied the mathematical model of Rabies by the concept of Caputo-Fabrizio fractional derivative. We solved the fractional differential equations by the Laplace Adomian decomposition method. The equilibrium points are calculated, and 
Table 3 Results of three types of derivative: Ordinary derivative $D^{\nu}$, Caputo fractional derivative ${ }^{c} D^{v}$, and Caputo-Fabrizio fractional derivative ${ }^{c f} D^{v}$ for $N(t)$

\begin{tabular}{lllllll}
\hline$t$ & 0 & 1 & 2 & 3 & 4 & 5 \\
\hline$D^{v}(v=1)$ & 10 & 10.19164 & 10.94056 & 12.24676 & 14.11024 & 16.531 \\
${ }^{c} D^{v}(v=0.95)$ & 10 & 10.2012980 & 10.951449 & 12.231931 & 14.033640 & 16.3503797 \\
${ }^{c f} D^{v}(v=0.95)$ & 9.998483 & 10.202680 & 10.942097 & 12.216736 & 14.026596 & 16.371678 \\
\hline
\end{tabular}

the existence and uniqueness of the solutions are studied by fixed point theorem. The stability of the method was investigated with the $F$-stability approach. Eventually, some numerical results are presented for different values of $\eta$ to show the effect of the fractional order. The effect of the model coefficients on the number of infected animals $I(t)$ has been investigated using figures, so that we observed that $b, c$, and $e$ have a direct effect on $I(t)$, whereas $a$ and $z$ have an inverse effect on $I(t)$. Finally, we compared the results of the integer-order derivative with the Caputo and Caputo-Fabrizio fractional-order derivatives. We observed that the behavior of $S(t), I(t), N(t)$ in all three types of derivatives is the same, but the resulting numerical values are different. Also, in some cases the results of the Caputo derivative are closer to those of integer-order derivative, and in some cases the results of the Caputo-Fabrizio derivative are closer to those of integer-order derivative. Therefore, with this information, it is not possible to conclude which type of fractional derivatives is better.

\section{Acknowledgements}

Research of the fourth author was supported by Azarbaijan Shahid Madani University. Also, research of the third author was supported by Miandoab Branch, Islamic Azad University. The authors are thankful to dear referees for their valuable comments which basically improved the final version of this work.

\section{Funding}

Not available.

Availability of data and materials

Data sharing not applicable to this paper as no datasets were generated or analyzed during the current study.

Ethics approval and consent to participate

Not applicable.

Competing interests

The authors declare that they have no competing interests.

Consent for publication

Not applicable.

Authors' contributions

The authors declare that the study was realized in collaboration with equal responsibility. All authors read and approved the final manuscript.

\section{Author details}

'Department of Mathematics, Istanbul Technical University, Ogretmenler Cad. 14 06530, Balgat, Istanbul, Turkey. ${ }^{2}$ Department of Mathematics, Cankaya University, Ankara, Turkey. ${ }^{3}$ Institute of Space Sciences, Magurele, Bucharest, Romania. ${ }^{4}$ Department of Mathematics, Miandoab Branch, Islamic Azad University, Miandoab, Iran. ${ }^{5}$ Institute of Research and Development, Duy Tan University, Da Nang, 550000, Vietnam. ${ }^{6}$ Faculty of Natural Sciences, Duy Tan University, Da Nang, 550000, Vietnam. ${ }^{7}$ Department of Medical Research, China Medical University Hospital, China Medical University, Taichung, Taiwan.

\section{Publisher's Note}

Springer Nature remains neutral with regard to jurisdictional claims in published maps and institutional affiliations. 


\section{References}

1. Kumar, V:: Robbins and Cotran Pathologic Basis of Disease. Elsevier, Saunders (2004)

2. Hassel, R., Vos, A., Clausen, P., Moore, S., der Westhuizen, J.V., Khaiseb, S., Kabajani, J., Pfaff, F., Höper, D., Hundt, B., Jago, M., Bruwer, F., Lindeque, P., Finke, S., Freuling, C.M., Muller, T.: Experimental screening studies on Rabies virus transmission and oral rabies vaccination of the greater kudu (tragelaphus strepsiceros). Sci. Rep. 8(1), 16599 (2018)

3. Meltzer, M.I., Rupprecht, C.E.: A review of the economics of the prevention and control of Rabies, part 1: global impact and Rabies in humans. PharmacoEcon. 14(4), 366-383 (1998)

4. Meltzer, M.I., Rupprecht, C.E.: A review of the economics of the prevention and control of Rabies, part 2: Rabies in dogs, livestock and wildlife. PharmacoEcon. 14(5), 481-498 (1998)

5. Demirci, E.: A new mathematical approach for Rabies endemy. Appl. Math. Sci. 8(2), 59-67 (2014)

6. Sterner, R.T., Smith, G.C.: Modelling wildlife Rabies. Biol. Conserv. 131(2), 163-179 (2006)

7. Zaeck, L., Potratz, M., Freuling, C.M., Muller, T., Finke, S.: High-resolution $3 d$ imaging of Rabies virus infection in solvent-cleared brain tissue. J. Vis. Exp. 146 (2019). https://doi.org/10.3791/59402

8. Alkahtani, B.S.T., Koca, I., Atangana, A.: Analysis of a new model of H1N1 spread: Model obtained via Mittag-Leffler function. Adv. Mech. Eng. 9(8), 1-8 (2017)

9. Buluta, H., Kumar, D., Singhb, J., Swroopc, R., Baskonus, H.M.: Analytic study for a fractional model of HIV infection of CD4+T lymphocyte cells. Math. Nat. Sci. 2, 33-43 (2018)

10. Dokuyucu, M.A., Celik, E., Bulut, H., Baskonus, H.M.: Cancer treatment model with the Caputop-Fabrizio fractional derivative. Eur. Phys. J. Plus 133, 92 (2018)

11. Koca, I.: Analysis of rubella disease model with non-local and non-singular fractional derivatives. Int. J. Optim. Control Theor. Appl. 8(1), 17-25 (2018)

12. Ucar, E., Ozdemir, N., Altun, E.: Fractional order model of immune cells influenced by cancer cells. Math. Model. Nat. Phenom. 14(3), 308 (2019)

13. Akbari Kojabad, E., Rezapour, S.: Approximate solutions of a sum-type fractional integro-differential equation by using Chebyshev and Legendre polynomials. Adv. Differ. Equ. 2017, 351 (2017)

14. Aydogan, M.S., Baleanu, D., Mousalou, A., Rezapour, S.: On high order fractional integro-differential equations including the Caputo-Fabrizio derivative. Bound. Value Probl. 2018(1), 90 (2018). https://doi.org/10.1186/s13661-018-1008-9

15. Aydogan, S.M., Baleanu, D., Mousalou, A., Rezapour, S.: On approximate solutions for two higher-order Caputo-Fabrizio fractional integro-differential equations. Adv. Differ. Equ. 2017(1), 221 (2017). https://doi.org/10.1186/s13662-017-1258-3

16. Baleanu, D., Agarwal, R.P., Mohammadi, H., Rezapour, S.: Some existence results for a nonlinear fractional differentia equation on partially ordered Banach spaces. Bound. Value Probl. 2013, 112 (2013)

17. Baleanu, D., Etemad, S., Pourrazi, S., Rezapour, S.: On the new fractional hybrid boundary value problems with three-point integral hybrid conditions. Adv. Differ. Equ. 2019, 473 (2019)

18. Baleanu, D., Guvenc, Z.B., Machado, J.A.T.: New Trends in Nano Technology and Fractional Calculus Applications Springer, Germany (2010)

19. Baleanu, D., Ghafarnezhad, K., Rezapour, S., Shabibi, M.: On the existence of solutions of a three steps crisis integro-differential equation. Adv. Differ. Equ. 2018, 135 (2018)

20. Baleanu, D., Ghafarnezhad, K., Rezapour, S.: On a three steps crisis integro-differential equation. Adv. Differ. Equ. 2019, $153(2019)$

21. Baleanu, D., Hedayati, V., Rezapour, S., Al-Qurashi, M.M.: On two fractional differential inclusions. SpringerPlus 5(1), 882 (2016)

22. Baleanu, D., Rezapour, S., Mohammadi, H.: Some existence results on nonlinear fractional differential equations. Philos. Trans. R. Soc. A, Math. Phys. Eng. Sci. 371, 20120144 (2013). https://doi.org/10.1098/rsta.2012.0144

23. Baleanu, D., Mohammadi, H., Rezapour, S.: On a nonlinear fractional differential equation on partially ordered metric spaces. Adv. Differ. Equ. 2013, 83 (2013)

24. Baleanu, D., Mohammadi, H., Rezapour, S.: The existence of solutions for a nonlinear mixed problem of singular fractional differential equations. Adv. Differ. Equ. 2013, 359 (2013)

25. Baleanu, D., Mousalou, A., Rezapour, S.: A new method for investigating approximate solutions of some fractional integro-differential equations involving the Caputo-Fabrizio derivative. Adv. Differ. Equ. 2017, 51 (2017)

26. Baleanu, D., Mousalou, A., Rezapour, S.: The extended fractional Caputo-Fabrizio derivative of order $0 \leq \sigma<1$ on $c 、[0,1]$ and the existence of solutions for two higher-order series-type differential equations. Adv. Differ. Equ. 2018, $255(2018)$

27. Baleanu, D., Mousalou, A., Rezapour, S.: On the existence of solutions for some infinite coefficient-symmetric Caputo-Fabrizio fractional integro-differential equations. Bound. Value Probl. 2017, 145 (2017)

28. Baleanu, D., Rezapour, S., Saberpour, Z.: On fractional integro-differential inclusions via the extended fractional Caputo-Fabrizio derivation. Bound. Value Probl. 2019, 79 (2019)

29. Ozdemir, N., Ucar, E.: Investigating of an immune system-cancer mathematical model with Mittag-Leffler kernel. AIMS Math. 5(2), 1519-1531 (2020). https://doi.org/10.3934/math.2020104

30. Yavuz, M., Ozdemir, N.: Analysis of an epidemic spreading model with exponential decay law. Math. Sci. Appl. E-Notes 8(1), 142-154 (2020). https://doi.org/10.36753/mathenot.6916384

31. Carvalho, A.R., Pinto, C.M., Tavares, J.N.: Maintenance of the latent reservoir by pyroptosis and superinfection in a fractional order HIV transmission model. Int. J. Optim. Control Theor. Appl. 9(3), 69-75 (2019). https://doi.org/10.11121/ijocta.01.2019.00643

32. Defterli, O.: Modeling the impact of temperature on fractional order Dengue model with vertical transmission. Int. J. Optim. Control Theor. Appl. 10(1), 85-93 (2020). https://doi.org/10.11121/ijocta.01.2020.00862

33. Ucar, S., Ozdemir, N., Koca, I., Altun, E.: Novel analysis of the fractional glucose-insulin regulatory system with non-singular kernel derivative. Eur. Phys. J. Plus 135, 414 (2020). https://doi.org/10.1140/epjp/s13360-020-00420-w

34. Talaee, M., Shabibi, M., Gilani, A., Rezapour, S.: On the existence of solutions for a pointwise defined multi-singular integro-differential equation with integral boundary condition. Adv. Differ. Equ. 2020, 41 (2020)

35. Caputo, M., Fabrizio, M.: A new definition of fractional derivative without singular kernel. Prog. Fract. Differ. Appl. 1(2), 73-85 (2015) 
36. Atangana, A., Alkahtani, B.S.T.: Analysis of the Keller-Segel model with a fractional derivative without singular kernel. Entropy 17(6), 4439-4453 (2015)

37. Erturk, V.S., Zaman, G., Momani, S.: A numeric analytic method for approximating a giving up smoking model containing fractional derivatives. Comput. Math. Appl. 64, 3068-3074 (2012)

38. Haq, F., Shah, K., Rahman, G., Shahzad, M.: Numerical analysis of fractional order model of HIV-1 infection of CD4+T-cells. Comput. Methods Differ. Equ. 5(1), 1-11 (2017)

39. Losada, J., Nieto, J.J.: Properties of the new fractional derivative without singular kernel. Prog. Fract. Differ. Appl. 1(2), 87-92 (2015)

40. Belgacem, F.B.M., Karaballi, A.A., Kalla, S.L.: Analytical investigations of the Sumudu transform and applications to integral production equations. Math. Probl. Eng. 3, 103-118 (2003)

41. Bodkhe, D.S., Panchal, S.K.: On Sumudu transform of fractional derivatives and its applications to fractional differential equations. Asian J. Math. Comput. Res. 11(1), 69-77 (2016)

42. Shah, K., Junaid, N.A.M.: Extraction of Laplace, Sumudu, Fourier and Mellin transform from the natural transform. J. Appl. Environ. Biol. Sci. 5(9), 1-10 (2015)

43. Watugala, G.K.: Sumudu transform: a new integral transform to solve differential equations and control engineering problems. Int. J. Math. Educ. Sci. Technol. 24(1), 35-43 (1993)

44. Wang, J., Zhou, Y., Medved, M.: Picard and weakly Picard operators technique for nonlinear differential equations in Banach spaces. J. Math. Anal. Appl. 389, 261-274 (2012)

45. Van den Driessche, P., Watmough, J.: Reproduction numbers and subthreshold endemic equilibria for compartmental models of disease transmission. Math. Biosci. 180, 29-48 (2002)

46. Li, H., Cheng, J., Li, H.B., Zhong, S.M.: Stability analysis of a fractional-order linear system described by the Caputo-Fabrizio derivative. Mathematics 7(2), 200 (2019)

47. Hosseini, M.M., Nasabzadeh, H.: On the convergence of Adomian decomposition method. Appl. Math. Comput. 182, 536-543 (2006)

48. Abdilrazec, A.H.M.: Adomian decomposition method: convergence analysis and numerical approximations. M.Sc. Dissertation, Mc Master University, Canada (2008)

49. Naghipour, A., Manafian, J.: Application of the Laplace Adomian decomposition method and implicit methods for solving Burgers equation. TWMS J. Pure Appl. Math. 6(1), 68-77 (2015)

\section{Submit your manuscript to a SpringerOpen ${ }^{\circ}$ journal and benefit from:}

- Convenient online submission

- Rigorous peer review

- Open access: articles freely available online

- High visibility within the field

- Retaining the copyright to your article

Submit your next manuscript at $>$ springeropen.com 